\title{
Radiological progression of cerebral metastases after radiosurgery: assessment of perfusion MRI for differentiating between necrosis and recurrence
}

\author{
Friso W. A. Hoefnagels · Frank J. Lagerwaard · \\ Esther Sanchez • Cornelis J. A. Haasbeek • \\ Dirk L. Knol · Ben J. Slotman · W. Peter Vandertop
}

Received: 26 May 2008/Revised: 3 November 2008/ Accepted: 19 November 2008/Published online: 10 March 2009

(C) The Author(s) 2009. This article is published with open access at Springerlink.com

\begin{abstract}
To assess the capability of perfusion MRI to differentiate between necrosis and tumor recurrence in patients showing radiological progression of cerebral metastases treated with stereotactic radiosurgery (SRS). From 2004 to 2006 dynamic susceptibility-weighted contrast-enhanced perfusion MRI scans were performed on patients with cerebral metastasis showing radiological progression after SRS during follow-up. Several perfusion MRI characteristics were examined: a subjective visual score of the relative cerebral blood volume ( $\mathrm{rCBV}$ ) map and quantitative rCBV measurements of the contrastenhanced areas of maximal perfusion. For a total of 34 lesions in 31 patients a perfusion MRI was performed. Diagnoses were based on histology, definite radiological decrease or a combination of radiological and clinical follow-up. The diagnosis of tumor recurrence was obtained in 20 of 34 lesions, and tumor necrosis in 14 of 34 . Regression analyses for all measures proved statistically significant $\left(\chi^{2}=11.6-21.6, \quad P<0.001-0.0001\right)$. Visual
\end{abstract}

F. W. A. Hoefnagels $(\varangle) \cdot$ W. Peter Vandertop Department Neurosurgery, VU University Medical Centre, P.O. Box 7057, 1007 MB Amsterdam, The Netherlands e-mail: f.hoefnagels@vumc.nl

F. J. Lagerwaard · C. J. A. Haasbeek · B. J. Slotman Department of Radiation Oncology, VU University Medical Center, Amsterdam, The Netherlands

E. Sanchez

Department of Radiology, VU University Medical Center, Amsterdam, The Netherlands

D. L. Knol

Department of Clinical Epidemiology and Biostatistics, VU University Medical Center, Amsterdam, The Netherlands inspection of the rCBV map yielded a sensitivity and specificity of 70.0 respectively $92.9 \%$. The optimal cutoff point for maximal tumor $\mathrm{rCBV}$ relative to white matter was 2.00 (improving the sensibility to $85.0 \%$ ) and 1.85 relative to grey matter (GM), improving the specificity to $100 \%$, with a corresponding sensitivity of $70.0 \%$. Perfusion MRI seems to be a useful tool in the differentiation of necrosis and tumor recurrence after SRS. For the patients displaying a rCBV-GM greater than 1.85 , the diagnosis of necrosis was excluded. Salvage treatment can be initiated for these patients in an attempt to prolong survival.

Keywords Cerebral metastases $\cdot$ Stereotactic radiosurgery $\cdot$ Perfusion MRI $\cdot$ Necrosis $\cdot$ Recurrence

\section{Introduction}

Cerebral metastases affect $20-30 \%$ of all cancer patients and form the second most common cerebral neoplasm in adults [1, 2]. Stereotactic radiosurgery (SRS), either as a single modality or in combination with whole brain radiotherapy (WBRT), is an established treatment option for patients with a limited number of brain metastases, increasing the mean overall survival rate from 3 to 6 months after WBRT to approximately $8-12$ months [1, $15,22,33,34]$. Radiation-induced changes on follow-up MRI studies have been reported in up to $22 \%$ of patients, and are frequently impossible to differentiate from local tumor progression [18, 35, 37, 39, 44, 50, 51]. In case of local tumor recurrence, surgical resection or repeat radiosurgery can be considered as salvage options for selected patients who are in good clinical condition and have stable or absent extracranial disease, underscoring the clinical relevance of this diagnostic dilemma. 
Histological verification of recurrence constitutes the gold standard, but this requires a resection of the lesion rather than biopsy for reasons of spatial heterogeneity, i.e., regions of viable tumor and necrosis may co-exist after SRS. Several non-invasive techniques, including SPECT, MR spectroscopy (HMRS) and PET, have been used to differentiate between radionecrosis and tumor progression [11, 17, 43, 47]. For FDG-PET, sensitivities between 65 and $86 \%$ (with MRI co-registration) and for HMRS and SPECT sensitivities and specificities around 85\%-90\% are reported, favoring SPECT [5, 11, 18, 19]. Besides the financial and labor-intensive aspects of these techniques, one of the major disadvantages is that the relatively low resolution might prevent (early) detection of recurrence in these heterogenous lesions [12]. Recent MRI techniques with higher spatial resolution have been suggested to yield better results [48, 52].

Dynamic susceptibility-weighted contrast-enhanced (DSC) or perfusion MRI is capable of quantifying microvessel density (vascularity) and permeability of brain tissue by assessment of the relative cerebral blood volume (rCBV) [3, 8, 10, 25, 27, 38, 46] and has been used for grading, histological differentiation and assessment of prognosis in glioma patients [6, 7, 16, 24, 29, 32, 54]. The literature on the use of DSC MRI for brain metastases is limited and mainly focuses on predicting treatment response [23, 48, 53].

The goal of this study is to evaluate the capability of perfusion MRI for differentiating between radiationinduced tumor necrosis and tumor progression in patients with radiosurgically treated cerebral metastases with progressive lesions during follow-up in the presence of salvage options.

\section{Methods and materials}

Patient population

At the VU University Medical Center Amsterdam, patients with one to three brain metastases have been treated with SRS as a single modality since 2002 . The SRS dose varied between 18 and $21 \mathrm{~Gy}$ prescribed at the $80 \%$ isodose, depending on the size of the treated lesion. The SRS-alone treatment option was combined with standardized MRI follow-up at 3-month intervals in order to allow for timely salvage therapy, when indicated. Those patients demonstrating a progression in volume of the treated lesion during follow-up and who were candidates for salvage therapy in the form of surgery or repeat SRS based on their general condition and extracranial tumor status routinely underwent a perfusion MRI to differentiate between radiationinduced tumor necrosis and tumor recurrence.
A total of 31 such patients harboring 34 treated lesions form the basis of this study. Relevant patient and treatment characteristics are shown in Table 1. The majority of patients (19/31) had metastases from lung cancer. The preradiosurgery metastasis volume ranged from 0.6 to $31.6 \mathrm{~cm}^{3}$ (mean $8.0 \mathrm{~cm}^{3}$ ). The mean volume after radiosurgery (prior to progression, i.e., the minimum volume measured during follow-up) was $3.2 \mathrm{~cm}^{3}$ (range $0.1-$ $10.7 \mathrm{~cm}^{3}$ ). The corresponding volume at the time of progression (and of the perfusion MRI) was $7.2 \mathrm{~cm}^{3}$ (range $0.2-25.3 \mathrm{~cm}^{3}$ ). The median SRS dose was $21 \mathrm{~Gy}$, prescribed at the $80 \%$ isodose. Four patients had received WBRT prior to SRS with a total dose of $20 \mathrm{~Gy}$ in five fractions of $4 \mathrm{~Gy}$.

\section{Clinical outcome}

Although histopathological verification is the gold standard in differentiating between tumor recurrence or radiationinduced tumor necrosis, this was available in only a small subgroup. In addition, some patients showed a substantial regression of lesion volume on subsequent follow-up MRI scans without additional treatment, making a diagnosis of tumor necrosis highly likely, and of tumor recurrence very unlikely. In all other patients, the clinical diagnosis was assessed subjectively using radiological and clinical followup data. A rapidly deteriorating neurological condition, short survival time due to neurological progression, or ongoing progression in subsequent MRI scans was considered indicative of tumor progression. All clinical diagnoses were defined retrospectively by the treating physician (FL), who was blinded to the perfusion MRI findings.

\section{MRI}

Imaging was performed on a 1.5-T MRI scanner (Siemens Sonata, Siemens Medical Systems, Erlangen, Germany). After a localizing sagittal T1-weighted image, nonenhanced axial T1-weighted spin echo [repetition time/ echo time (TR/TE) 600/12 ms] and axial T2-weighted $(3,680 / 85)$ images were obtained. Post-contrast axial and sagittal (MPR) T1-weighted imaging was performed after the acquisition of the DSC MRI data.

DSC MRI scans were acquired with a gradient-echo echoplanar imaging (GE-EPI) technique during the first pass of a standard dose $(0.1 \mathrm{mmol} / \mathrm{kg})$ bolus of gadolinium contrast (Magnevist; Berlex Laboratories). Imaging parameters were: TR/TE 1,440/47; slice thickness $5 \mathrm{~mm}$, interslice gap $1.5 \mathrm{~mm}$, field of view 230-230 mm, matrix 128-128 leading to pixel size $0.9-0.9 \mathrm{~mm}$ (after interpolation), flip angle $90^{\circ}$, and signal bandwidth, 1,345 Hz/pixel. The injection rate of the contrast was $5 \mathrm{ml} / \mathrm{s}$. A total of 50 images were acquired at 1.44-s intervals with the bolus typically arriving between the 
Table 1 Patient characteristics and perfusion measures

\begin{tabular}{|c|c|c|c|c|c|c|c|c|c|c|c|c|c|}
\hline & \multicolumn{2}{|c|}{ Patient } & \multirow{2}{*}{$\begin{array}{l}\text { Metastasis } \\
\text { Prim. tumor }\end{array}$} & \multicolumn{3}{|c|}{ Initial prior } & \multirow[t]{2}{*}{ TTP } & \multirow[t]{2}{*}{ PPS } & \multirow[t]{2}{*}{ OS } & \multirow{2}{*}{$\begin{array}{l}\text { Clinical } \\
\text { Diagnosis }\end{array}$} & \multicolumn{2}{|l|}{$\mathrm{rCBV}$} & \multirow{2}{*}{$\begin{array}{l}\text { rCBV map } \\
\text { Diagnosis }\end{array}$} \\
\hline & Sex & Age & & Vol (cc) & Dose & Therapy & & & & & WM & GM & \\
\hline 1 & M & 54 & Lung & 19.8 & 24 & None & 10 & 6.5 & 16.8 & Necrosis & 1.57 & 0.62 & Necrosis \\
\hline 2 & M & 68 & Lung & 10.5 & 21 & $\mathrm{NSR}+\mathrm{RTH}$ & 4 & 1 & 5.2 & Progression & 2.31 & 0.99 & Progression \\
\hline 3 & M & 57 & Lung & 1.1 & 21 & $\mathrm{BPY}+\mathrm{RTH}$ & 8.5 & 16.5 & 25.2 & Progression* & 4.69 & 2.66 & Progression \\
\hline 4 & $\mathrm{~F}$ & 66 & Lung & 7.1 & 24 & None & 6 & 2 & 8.2 & Necrosis & 0.94 & 0.48 & Necrosis \\
\hline 5 & M & 43 & Lung & 5.8 & 18 & NSR & 3 & 17.5 & 20.6 & Progression* & 6.72 & 5.16 & Progression \\
\hline 6 & $\mathrm{~F}$ & 47 & Lung & 3.0 & 21 & None & 17 & 20 & 36.6 & Necrosis $^{\mathrm{a}}$ & 1.50 & 0.78 & Non concl. \\
\hline $7 \mathrm{a}$ & M & 44 & Lung & 13.6 & 18 & None & 12 & 12 & 23.9 & Progression & 4.15 & 1.99 & Progression \\
\hline $7 b$ & M & 44 & Lung & 2.6 & 18 & None & 12 & 12 & 23.9 & Necrosis $^{\mathrm{a}}$ & 3.87 & 1.85 & Non concl. \\
\hline 8 & M & 43 & Lung & 3.8 & 21 & None & 7.5 & 6 & 13.7 & Necrosis & 0.94 & 0.47 & Non concl. \\
\hline 9 & $\mathrm{~F}$ & 56 & Lung & 18.9 & 24 & RTH & 11 & 9 & 20.0 & Progression & 4.73 & 3.49 & Progression \\
\hline 10 & $\mathrm{~F}$ & 58 & Lung & 5.8 & 18 & None & 9 & 20 & 29.2 & Necrosis $^{\mathrm{a}}$ & 1.64 & 0.60 & Necrosis \\
\hline 11 & $\mathrm{~F}$ & 72 & Lung & 9.2 & 21 & None & 3.5 & 1.5 & 4.9 & Progression & 0.97 & 0.55 & Necrosis \\
\hline 12 & $\mathrm{~F}$ & 47 & Lung & 6.4 & 18 & None & 7.5 & 20.5 & 28.0 & Necrosis $^{\mathrm{a}}$ & 3.09 & 0.59 & Necrosis \\
\hline 13 & M & 45 & Lung & 13.0 & 24 & None & 7 & 4.5 & 11.9 & Necrosis & 1.98 & 1.09 & Necrosis \\
\hline 14 & $\mathrm{~F}$ & 72 & Lung & 1.7 & 15 & None & 7.5 & 11.5 & 19.3 & Necrosis & 3.71 & 1.47 & Necrosis \\
\hline 15 & M & 58 & Lung & 8.4 & 18 & RTH & 6 & 1.5 & 7.6 & Progression & 2.04 & 1.95 & Necrosis \\
\hline 16 & $\mathrm{~F}$ & 57 & Lung & 0.9 & 21 & None & 7.5 & 3 & 10.3 & Progression* & 2.80 & 0.78 & Necrosis \\
\hline 17 & $\mathrm{~F}$ & 62 & Lung & 10.7 & 18 & None & 6.5 & 7 & 13.5 & Progression & 3.90 & 2.29 & Progression \\
\hline 18 & $\mathrm{~F}$ & 41 & Lung & 12.7 & 24 & None & 4 & 6.5 & 10.5 & Progression* & 4.67 & 2.22 & Progression \\
\hline 19 & M & 70 & Lung & 3.9 & 18 & None & 8.5 & 5 & 13.6 & Progression & 2.58 & 1.45 & Non concl. \\
\hline 20 & $\mathrm{~F}$ & 51 & Mamma & 9.1 & 18 & None & 9.5 & 7 & 16.8 & Progression & 4.19 & 2.32 & Progression \\
\hline 21 & $\mathrm{~F}$ & 36 & Mamma & 10.6 & 18 & NSR & 11.5 & 12.5 & 24.2 & Necrosis & 2.31 & 1.33 & Necrosis \\
\hline 22 & $\mathrm{~F}$ & 51 & Mamma & 8.1 & 18 & None & 7 & 22 & 28.8 & Progression* & 13.69 & 5.74 & Progression \\
\hline 23 & $\mathrm{~F}$ & 36 & Mamma & 1.3 & 15 & None & 7 & 28 & 35.5 & Necrosis $^{\mathrm{a}}$ & 1.24 & 0.92 & Non concl. \\
\hline 24 & $\mathrm{~F}$ & 46 & Mamma & 0.6 & 21 & NSR & 15 & 24 & 38.6 & Progression & 6.22 & 2.67 & Progression \\
\hline $25 \mathrm{a}$ & $\mathrm{F}$ & 54 & Ovary & 4.1 & 18 & None & 7.5 & 4 & 11.8 & Progression & 2.40 & 1.86 & Progression \\
\hline $25 b$ & $\mathrm{~F}$ & 54 & Ovary & 1.2 & 18 & None & 7.5 & 4 & 11.8 & Progression* & 5.86 & 4.54 & Progression \\
\hline $25 \mathrm{c}$ & $\mathrm{F}$ & 54 & Ovary & 15.4 & 18 & None & 7.5 & 4 & 11.8 & Progression & 1.23 & 0.95 & Necrosis \\
\hline 26 & M & 53 & Melanoma & 20.8 & 24 & None & 9 & 5.5 & 14.4 & Progression & 7.84 & 4.66 & Progression \\
\hline 27 & $\mathrm{~F}$ & 62 & Melanoma & 3.3 & 21 & None & 12 & 1 & 12.9 & Progression & 0.75 & 0.31 & Necrosis \\
\hline 28 & $\mathrm{~F}$ & 60 & Renal cell & 3.8 & 21 & None & 7.5 & 3 & 10.6 & Necrosis & 1.56 & 1.17 & Progression \\
\hline 29 & M & 59 & Renal cell & 3.2 & 21 & None & 7 & 7.5 & 14.7 & Progression & 4.21 & 2.77 & Progression \\
\hline 30 & M & 49 & Bladder & 31.6 & 24 & None & 9.5 & 3.5 & 13.2 & Necrosis & 0.55 & 0.55 & Non concl. \\
\hline 31 & $\mathrm{~F}$ & 56 & Colon & 0.9 & 21 & None & 8 & 16 & 23.9 & Necrosis & 0.47 & 0.29 & Necrosis \\
\hline
\end{tabular}

The first columns show the most important patient characteristics; the last three show the different perfusion parameters. Age is in years; dose is radiation dose in Grays at the $80 \%$ isodose center; survival times are in months. NSR neurosurgical resection, $B P Y$ biopsy, $R T H$ radiotherapy, $T T P$ time to (radiological) progression, $O S$ overall survival time, $P P S$ survival time after radiological progression. $r C B V$ relative cerebral blood volume, WM white matter, GM grey matter, non concl non-conclusive. *Diagnosis based on histology, adiagnosis based on radiological decrease at follow-up

10th and 15th image. The post-processing of the DSC MRI data were performed on a Leonardo VD10B Syngo OEM installation (Siemens AG).

DSC measurements

The DSC MRI scans were scored based on both subjective and objective evaluations of the rCBV maps by an experienced neuroradiologist (E.S.), who was blinded with respect to the clinical information and outcome.

\section{Subjective scoring of the $r C B V$ maps}

The rCBV map was evaluated alongside the post-contrast structural MRI series. A subjective diagnosis of "tumor progression" was based on the presence of nodular highly 
vascularized areas within the contrast-enhanced lesion, irrespective of areas indicative of necrosis (Fig. 1a), relative to the contralateral hemisphere. Alternatively, a diagnosis of "tumor necrosis" was made in case of a clear absence of perfusion (black hole), in the absence of any nodular highly vascularized area (Fig. 1b). When neither a high perfusion area nor a clear perfusion absence could be determined, the rCBV map was scored as "non-conclusive."
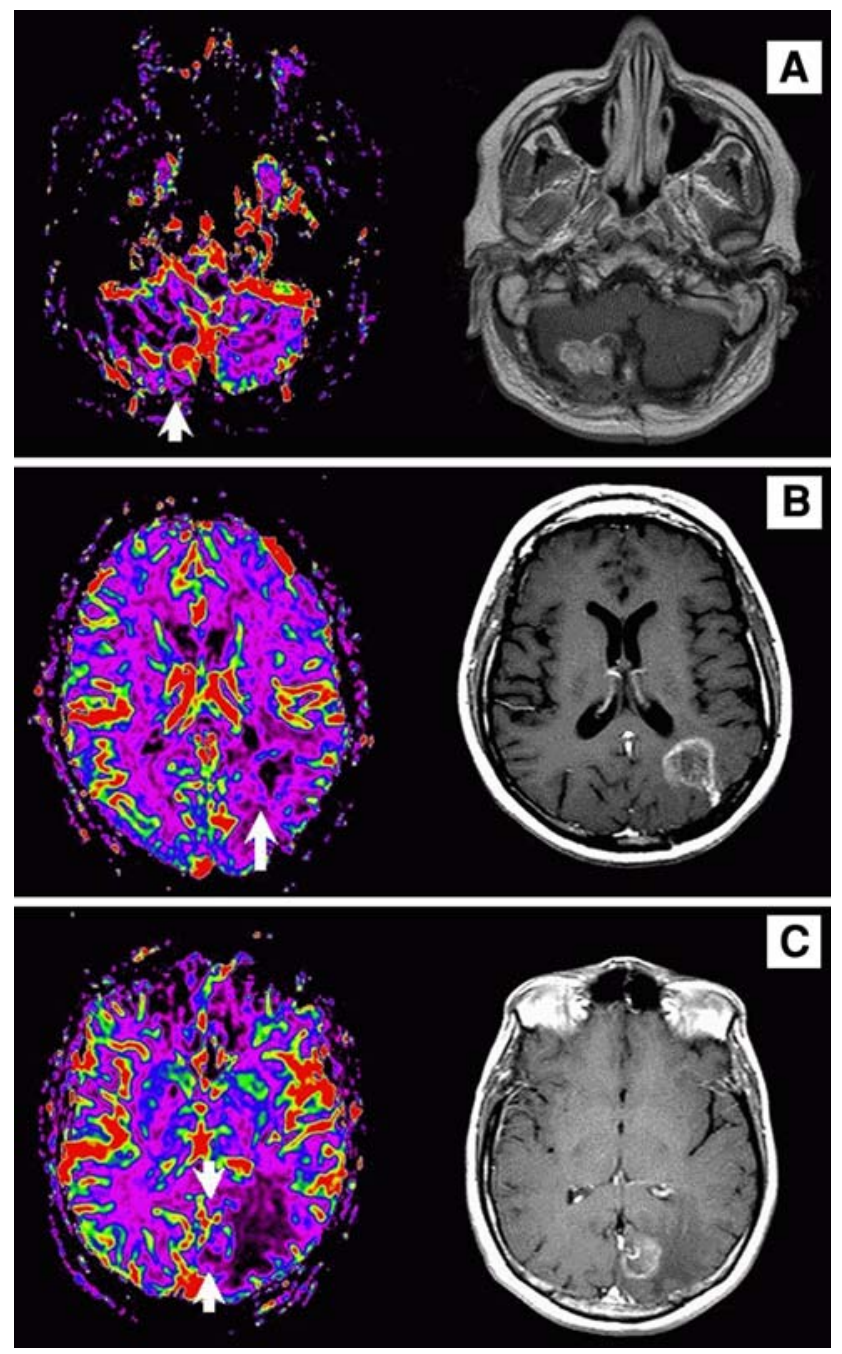

Fig. 1 rCBV maps and T1-weighted scans after gadolinium administration. Patient A shows a clear high $\mathrm{rCBV}$ at the site of the contrastenhanced lesion (Fig. 1a, arrow), suggestive of tumor recurrence. The lesion was resected, and the histological diagnosis was tumor recurrence. Patient B displays a perfusion absence (Fig. 1b, "black spot," arrow), suggestive of necrosis, at the lesion site as indicated by the post-contrast scan. This was confirmed by clinical and radiological follow-up. For the last patient (C) the rCBV map was judged "inconclusive," since no clear high CBV nor a clear absence thereof (between arrows) was present at the site of enhancement. The clinical diagnosis based on continued clinical and radiological follow-up was necrosis

\section{$r C B V$ measurements}

The procedure used to calculate rCBV from DSC MRI data is based on standard algorithms. The principles of calculating rCBV from signal intensity curves during the first pass of a bolus contrast agent have been described extensively by others $[14,20]$. Since spatial heterogeneity is a possible confounding factor in recurrent lesions, rCBVs were calculated for the highest perfusion fraction, irrespective of whether that area was judged as being actually high relative to contralateral white or grey matter, in concordance with previous reports [24, 26, 55]. In addition, as irradiated tumor rCBVs typically lie between white matter and grey matter values [17], a mean grey matter CBV, calculated from three regions Of interest (ROIs) spread over the contralateral cortex (one frontal, one temporal and one occipital) after exclusion of large vessels to minimize the chances of partial volume of white matter voxels [28] was used as a reference region in addition to a contralateral white matter reference region (semioval center). Thus, two quantitative parameters were calculated: $\mathrm{rCBV}-\mathrm{WM}$ and rCBV-GM.

\section{Statistical methods}

Kaplan-Meyer statistics and the log-rank test were used for a survival analysis of the different clinical diagnosis groups. Logistic regression models were fitted for evaluation of the value of the subjective and objective rCBV map conclusions for predicting the clinical diagnosis. For all objective/quantitative measures, ROC curves were plotted to calculate the most discriminative cutoff point with an optimal area under the curve and to define the clinically most relevant combination of sensitivity, specificity and accuracy. All statistical evaluations were computed using SPSS 12.0.1 for Windows, released November 2003, Copyright @ SPSS Inc., 1989-2003.

\section{Results}

\section{Clinical outcome}

In 6 of the 34 lesions (18\%), a histological diagnosis was obtained (1 post-mortem), and all showed viable tumor on pathological examination. Five lesions (15\%) showed subsequent spontaneous radiological regression without treatment, indicative of tumor necrosis. Thus, an objective outcome parameter could be obtained for 11 lesions (35\%). For the remaining 23 lesions, the clinical diagnosis was assigned as tumor recurrence in 14 lesions and tumor necrosis in 9 lesions, based retrospectively on radiological follow-up and clinical course. In total, 20 lesions (59\%) 
were assigned to the recurrence group and 14 lesions (41\%) to the tumor necrosis group (see Fig. 2).

The mean time to radiological progression, i.e., the interval between SRS and lesion enlargement on follow-up MRI scans, was not significantly different between the two groups: $9.2 \pm 2.8$ months for the tumor necrosis group and $7.8 \pm 3.0$ months for the tumor progression group $(P=0.21)$. Although the mean survival calculated from the time of suspected recurrence (time of perfusion MRI) was longer for the patients scored as having tumor necrosis (13.4 \pm 8.6 months) than for the tumor recurrence patients (8.4 \pm 7.1 months), this difference did not reach statistical significance $(P=0.08)$, see Fig. 3 (left Kaplan-Meier curve). However, 8 of the 20 patients with tumor recurrence received adjuvant treatment (5 patients underwent neurosurgical resection, and 3 patients received radiotherapy) and had a mean survival from time of radiological progression of 12.8 months, against a mean survival of 5.4 months in the remaining patients, thereby prolonging the survival of the total recurrence group. The mean overall survival for the entire cohort (all 31 patients) is remarkably long: 18.8 months from the moment of initial SRS.

\section{Subjective scoring of the rCBV map}

Fifteen of the 34 lesions were classified as tumor recurrence (Fig. 1a), and 13 lesions were diagnosed as radiation necrosis (Fig. 1b). This left six lesions for which the rCBV map was considered non-conclusive (Fig. 1c) (Table 1). Logistic regression analysis was performed to calculate how well the rCBV map diagnosis in itself is capable of predicting actual clinical tumor recurrence, and this showed a sensitivity and specificity of 70.0 and $92.9 \%$,

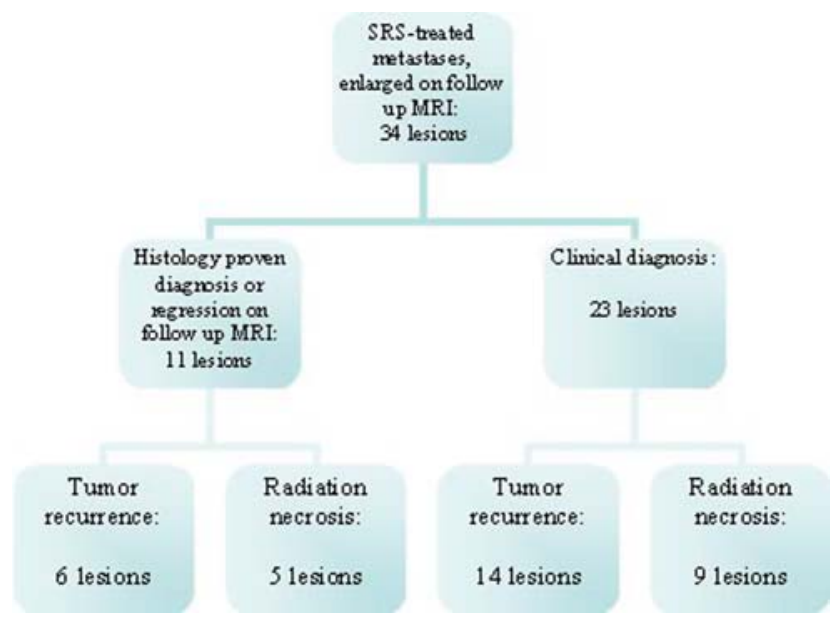

Fig. 2 Distribution of diagnoses across lesions. In total, 20 lesions were considered to be tumor progression (6 with a histologically confirmed diagnosis and 14 with a clinical diagnosis) and 14 to be radiation necrosis (5 regressing lesions on further follow-up and 9 based on clinical diagnoses)

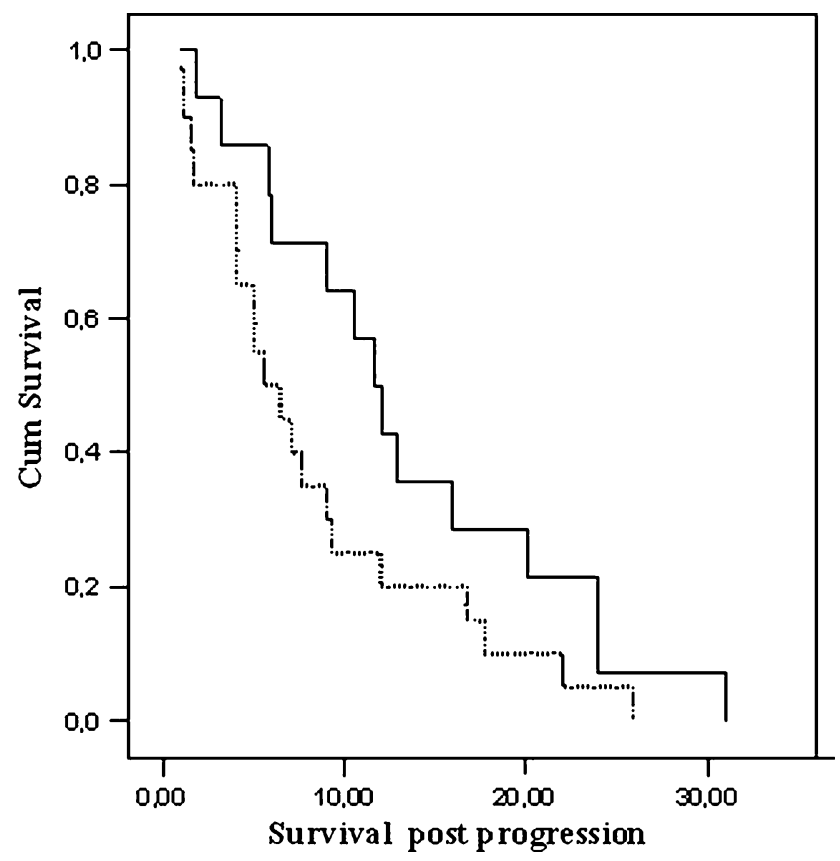

Fig. 3 Kaplan-Meier survival curves. The curve shows no significant survival between the tumor progression (dotted line) and radiation necrosis group (straight line); survival in months, after radiological enlargement of the stereotactically irradiated lesion

respectively $\left(\chi^{2}=15.99 ; \mathrm{df}=2 ; P<0.001 ; R^{2} 0.506\right)$, and positive (PPV) and negative predictive values (NPV) of $93.3 \%$ and $68.4 \%$, respectively. Of the six dubious lesions (either because of a faint ring around the lesion on the $\mathrm{rCBV}$ map or the absence of a clear necrotic/very low perfusion area) for which no diagnosis could be obtained by subjective scoring of the rCBV map, five were judged radiation effects, and just one was considered tumor progression, based on clinical outcome.

\section{Quantitative rCBV measures}

The mean rCBV of the recurrence group was significantly larger than that of the tumor necrosis group for both rCBV$\mathrm{WM}$ and $\mathrm{rCBV}-\mathrm{GM}$ (mean differences of -2.5 respectively -1.6 , see Table 2). Logistic regression analysis yielded statistically significant models for both rCBV measures, favoring rCBVmax-GM, with a fairly good sensitivity and specificity ranging from 71.4 to $80.0 \%$ (see Table 3). ROC curves, plotted for both measures (Fig. 4 and Table 3) to demarcate the optimal cutoff point and corresponding rCBV value, yielded cutoff points of 2.00 and 1.85 . This resulted in a sensitivity of $85 \%$ and corresponding specificity of $71.4 \%$ for rCBVmax-WM and a $100 \%$ specificity with corresponding sensitivity of $70 \%$ for rCBVmax-GM (Table 2). In other words, all lesions displaying a maximal rCBV higher than 1.85 relative to grey matter could be identified as tumor recurrence. 
Table 2 rCBV means and group differences

\begin{tabular}{llllll}
\hline Variable & Necrosis $(n=14)$ & Progression $(n=20)$ & $t$ & $P$-value & $95 \%$ CI \\
\hline rCBV-high-WM & 1.8123 & 4.2982 & -3.459 & 0.002 & $(-3.96-1.01)$ \\
rCBV-high-GM & 0.8723 & 2.4672 & -4.336 & $<0.001$ & $(-2.36-0.84)$ \\
\hline
\end{tabular}

Table 3 Results of logistic regression analysis (univariate)

\begin{tabular}{|c|c|c|c|c|c|c|}
\hline Variable & $\chi^{2 *}$ & Sign. & Nagelkerke $R^{2}$ & Sensitivity (\%) & Specificity (\%) & Accuracy (\%) \\
\hline \multicolumn{7}{|l|}{ All lesions } \\
\hline rCBV map & 15.992 & $<0.001$ & 0.506 & 70.0 & 92.9 & 79.4 \\
\hline rCBV-high-WM & 11.897 & 0.001 & 0.398 & 80.0 & 71.4 & 76.5 \\
\hline rCBV-high-GM & 15.697 & $<0.001$ & 0.498 & 75.0 & 78.6 & 76.5 \\
\hline rCBVmax-GM $>1.85$ & 21.635 & $<0.001$ & 0.634 & 70.0 & 100.0 & 82.4 \\
\hline rCBVmax-WM $>2.00$ & 11.574 & 0.001 & 0.389 & 85.0 & 71.4 & 79.4 \\
\hline \multicolumn{7}{|l|}{ Certain diagnosis } \\
\hline rCBV map & 11.339 & 0.003 & 0.86 & 83.3 & 100.0 & 90.9 \\
\hline rCBV-high-WM & 8.604 & 0.003 & 0.725 & 83.3 & 80.0 & 81.8 \\
\hline rCBV-high-GM & 7.526 & 0.006 & 0.663 & 83.3 & 80.0 & 81.8 \\
\hline rCBVmax-GM > 1.85 & 9.751 & 0.002 & 0.786 & 83.3 & 100.0 & 90.9 \\
\hline rCBVmax-WM $>2.00$ & 6.161 & 0.013 & 0.573 & 100.0 & 60.0 & 81.8 \\
\hline
\end{tabular}

Results are shown for several parameters. First the rCBV map diagnosis and the quantitative measures rCBV-high-WM and -GM, representing the highest perfusion fraction relative to white and grey matter, were separately entered. Next, the procedure was repeated for the high rCBV measures at their most discriminative cutoffs (1.85 and 2.0 relative to contralateral grey matter and white matter, respectively), according to the ROC curve analysis (see Fig. 4), thereby dichotomizing the groups. * $\mathrm{df}=2$ for $\mathrm{rCBV}$ map and $\mathrm{df}=1$ for all other variables

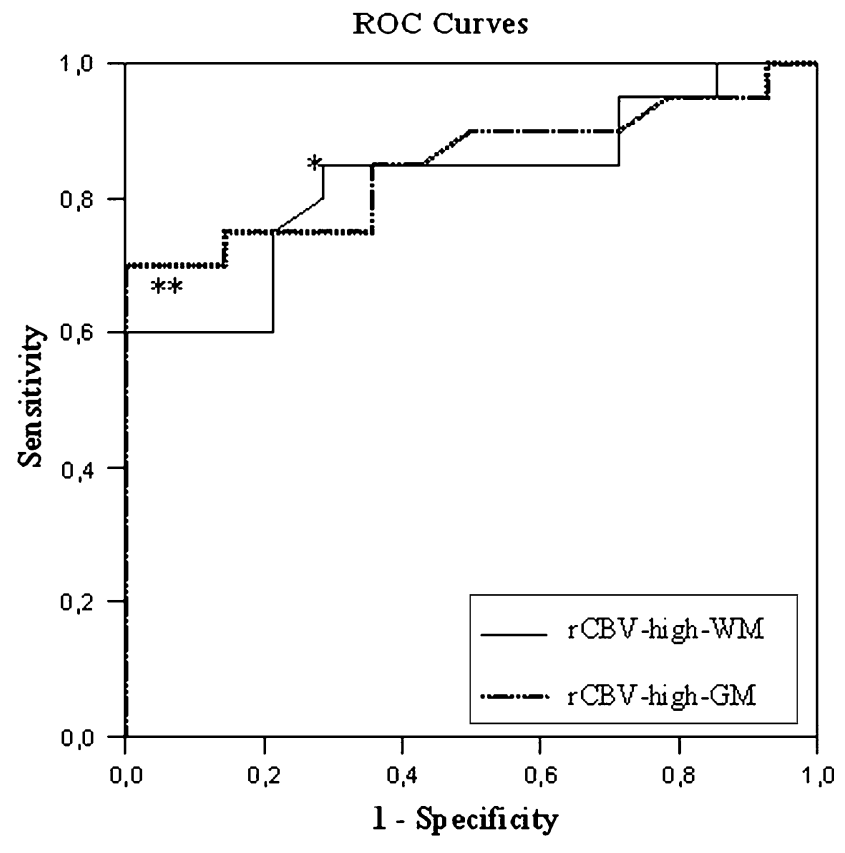

Fig. 4 ROC curves. The figure displays the ROC curves for rCBVhigh-WM (straight line) and -GM (dotted line), with areas under curves of 0.827 and 0.839 , respectively. The most discriminative cutoff point for rCBV-high-WM $(*)$ is 2.01 with a sensitivity of $85.0 \%$ and specificity of $71.4 \%$. For rCBV-high-GM, this point $(* *)$ corresponds to a cutoff of 1.85 with a sensitivity of $70.0 \%$ and specificity of $100 \%$
When the subgroup of patients with a histologically confirmed diagnosis or with spontaneous tumor regression on follow-up imaging was evaluated, analysis of the perfusion parameters demonstrated even better results with accuracies ranging from 81.8 to $90.9 \%$ (Table 3). Using a cutoff value of 2.0 for the rCBVmax_WM, a sensitivity of $100 \%$ was reached, and likewise a specificity of $100 \%$ when a rCBVmax_GM higher than 1.85 was chosen. Despite these remarkable results and even distribution, the total number of patients is considered too small to draw any firm conclusions.

\section{Discussion}

Enlargement of metastatic lesions after SRS, suggesting either tumor recurrence or necrosis and affecting 20-28\% of all treated lesions, constitutes a clinically relevant diagnostic dilemma in patients in good functional condition and with stable extracranial disease. The long mean overall survival in this study of 18.8 months after SRS illustrates this fact. In order to prevent unnecessary neurosurgical interventions or expensive, labour-intensive and often inconclusive imaging techniques, the efficacy of perfusion MRI to differentiate between tumor necrosis and recurrence 
was evaluated. In our series, the detection of a nodular fractional high perfusion area on the $\mathrm{rCBV}$ map (within the contrast-enhanced lesion) or a maximum rCBV higher than 1.85 relative to grey matter was indicative of tumor recurrence, with a specificity of $100 \%$ and sensitivity of $70.0 \%$. In a small subgroup of patients with "certain" diagnoses, these results even improved (i.e., the sensitivity), which points to a possible underestimation of its recurrence detection potential.

\section{Current imaging modalities}

Traditionally, PET, SPECT and (lately) H-MR spectroscopy have been used to evaluate the 'tumor recurrenceradiation-induced necrosis' dilemma. In general (FDG)PET is probably the least sensitive, although better results are reported for high grade gliomas than for metastases, for which sensitivities between 65 and $80 \%$ are reported, with an improvement to $86 \%$ with MRI co-registration [5, 11, 13]. The use of ${ }^{11} \mathrm{C}$-methionine-tracers seems to improve these results somewhat $[45,49]$. The results for HMRS and SPECT are variable [4, 13, 30, 36, 40-42, 45], with most papers reporting the superiority of SPECT (sensitivity and specificity around $90-100 \%$ ), but again mainly in high grade gliomas. The results drop to sensitivities between 85 and $91 \%$ when metastases only are considered, unfortunately usually without histological diagnosis [19, 43].

In particular, the pitfall of frequent false-positive findings that are obtained with these techniques remains a matter of concern [39], as this may results in unnecessary interventions with associated morbidity or mortality. The advantage of the method we used is the high specificity, excluding false-positive results, and the better resolution than on Spect, HMRS and PfalseET, since $90 \%$ of the resected SRS-treated recurrent metastases are known to demonstrate a mixture of tumor proliferation and necrosis [48].

Metastasis and perfusion MRI: rationale and literature

The blood volume in tumor progression (represented by the rCBV) is increased as a consequence of a combination of intravascular and extravascular components: neocapillary formation, dilatation of existing vasculature and high permeability of metastatic tumor vessels [3, 8-10, 25]. Although the contrast enhancement of radiation effects is also a consequence of increased vascular permeability, there is a clear difference between the vascular dynamics of this increase compared to tumor progression. The transport of blood (contrast) into necrotic areas is a consequence of slow permeability limited by the diffusion distance (not measured by rCBV), whereas the fast permeability seen in (metastatic) viable tissue is determined by vessel permeability, which is partially responsible for the increased blood volume [31]. Areas with radiation-induced necrosis, on the contrary, do not show an increased intravascular blood volume. Hence, contrast enhancement, which is an indicator of integrity of the blood-brain-barrier, is not equivalent to the perfusion abnormalities measured by the $\mathrm{rCBV}$, which measures vascularity and might therefore differentiate between necrosis and progression [20, 25].

Previous reports on SRS-treated metastases have demonstrated that temporal changes in perfusion MRI measurements are useful for differentiating radiological responders from non-responders [23, 53]. However, in these studies no differentiation was made between tumor necrosis and recurrence. Others have differentiated between these two diagnoses using perfusion MRI; however, this was only for (high grade) gliomas [17, 47]. Truong et al. used perfusion MRI in the evaluation of progressive metastases after SRS and found a reasonable positive predictive value of $80 \%$ in a subgroup of patients (15/38). Although they obtained a histological diagnosis in all patients, in contrast to our study, their measurements were not quantified, and no true-negative lesions were reported, despite two false-negative lesions [48]. Our results are in agreement with the results of Koichi et al. [21], who found the same cutoff value of 2.0 relative to WM for differentiating necrosis from tumor recurrence after SRS.

\section{Perfusion MRI sensitivity}

A substantial portion of the progressive metastases possibly remain undetected given the calculated sensitivity. Part of this underestimation might be due to an incorrect clinical diagnosis since a considerably higher sensitivity and larger mean difference between both groups were found in the "certain diagnosis" analysis. Alternatively, since the rCBV values of treated enhancing tumor lie between the rCBV of white and grey matter, as stated by Henry et al., the assessment of cortical lesions is problematic, and relatively high rCBVs are needed for a clear diagnosis of tumor progression, thereby challenging overall sensitivity [17]. The regional cerebral blood volume (rCBV) of white matter (especially when edema is present) is low compared to the rCBV of grey matter, and the rCBV of treated lesions in this study often showed intermediate values. Any (small) increase in $\mathrm{rCBV}$ of treated lesions, e.g., due to radiationinduced or inflammatory reactions of surrounding tissues, will have a relatively higher impact on the ratio $\mathrm{rCBV}$ lesion/rCBV-WM than on the rCBV-lesion/rCBV-GM. This could lead to a false-positive labeling of lesions as 'progression' based on rCBV-WM.

Secondly, lesions are often located on the junction of grey and white matter. Since the rCBV map has a lower 
resolution than the conventional MRI, controversy might arise whether the area of enhanced perfusion is due to a progression of the metastasis in the white matter, or surrounding grey matter. When the $\mathrm{rCBV}$ is higher than the grey matter, the odds are that the high perfusion indeed indicates progressive disease. On the other hand, one could speculate that initial tumor recurrence is possibly independent of increasing vasculature, and therefore, a relatively early perfusion scan might miss the correct diagnosis. In this respect, others have found serial perfusion MRI more accurate in the evaluation of radiated metastases [23, 53].

\section{Limitations}

Several limitations of the present study need to be addressed. First, and most importantly, in only a small subgroup of the patients (18\%) a definite histological diagnosis was established. This is however inherent to the clinical dilemma, since the majority of patients present with an asymptomatic radiological enlargement during routine follow-up. For these patients with a high probability of radiation effects, the risks of possible complications of a resection or biopsy was deemed to outbalance the benefits of a confirmative tissue diagnosis. The volumetric decrease during further follow-up in a significant proportion of the patients together with the fact that no cases of radiation necrosis were demonstrated among the operated patients seems to justify this wait-and-scan policy.

The authors of this study felt that judgment of the clinical course until death, or at least 5 months after performing the perfusion scan, combined with frequent radiological evaluation justified the assignment to a diagnosis group in the absence of histological confirmation. Our results obtained through a clinical diagnosis have led to a possible underestimation of the capacity of perfusion MRI to differentiate between both entities, since better results were achieved for the "certain diagnosis" subgroup.

Secondly, our conclusions are based on a rather limited number of patients. The group is however homogenous, with a clinically relevant dilemma, and all data were gathered through a consistent protocol for all patients. We are continuing to perform perfusion MRI scans in patients with enlarging lesions during follow-up after radiosurgery for brain metastases, and will update the findings in due course. Finally, since perfusion MRI was done on indication (i.e., after progressive contrast enhancement), not all perfusion scans were achieved at the same time point after SRS. Theoretically this poses the problem of introducing early transient radiation effects, which occur slightly earlier (within 3 months) than tumor recurrence or radiation necrosis [18]. However, all perfusion scans in this study were performed at least 3 months after SRS (mean
8.4 months), thereby excluding possible contamination with transient radiation effects.

\section{Conclusion}

In conclusion, DSC MRI appears to be a useful instrument to differentiate between tumor recurrence and radiation necrosis after stereotactically irradiated cerebral metastases. Based on our data, a cutoff rCBV value higher than 1.85 relative to gray matter can be used to diagnose tumor progression, as no patient with radionecrosis had values exceeding this value. However, a value lower than 1.85 cannot be used for diagnosing radionecrosis, since some patients with (eventual) progressive disease displayed lower values. To further improve the sensitivity of this modality, future research is needed to elucidate a potential benefit of repeated perfusion MRI measurements possibly in conjunction with other imaging modalities.

Conflict of interest notification No actual or potential conflict of interest exists.

Open Access This article is distributed under the terms of the Creative Commons Attribution Noncommercial License which permits any noncommercial use, distribution, and reproduction in any medium, provided the original author(s) and source are credited.

\section{References}

1. Andrews DW, Scott CB, Sperduto PW, Flanders AE, Gaspar LE, Schell MC et al (2004) Whole brain radiation therapy with or without stereotactic radiosurgery boost for patients with one to three brain metastases: phase III results of the RTOG 9508 randomised trial. Lancet 363:1665-1672

2. Aoyama H, Shirato H, Tago M, Nakagawa K, Toyoda T, Hatano $\mathrm{K}$ et al (2006) Stereotactic radiosurgery plus whole-brain radiation therapy vs stereotactic radiosurgery alone for treatment of brain metastases: a randomized controlled trial. JAMA 295:2483-2491

3. Aronen HJ, Gazit IE, Louis DN, Buchbinder BR, Pardo FS, Weisskoff RM et al (1994) Cerebral blood volume maps of gliomas: comparison with tumor grade and histologic findings. Radiology 191:41-51

4. Bader JB, Samnick S, Moringlane JR, Feiden W, Schaefer A, Kremp S et al (1999) Evaluation of 1-3-[123I]iodo-alpha-methyltyrosine SPET and [18F]fluorodeoxyglucose PET in the detection and grading of recurrences in patients pretreated for gliomas at follow-up: a comparative study with stereotactic biopsy. Eur J Nucl Med 26:144-151

5. Belohlavek O, Simonova G, Kantorova I, Novotny J Jr, Liscak R (2003) Brain metastases after stereotactic radiosurgery using the Leksell gamma knife: can FDG PET help to differentiate radionecrosis from tumour progression? Eur J Nucl Med Mol Imaging 30:96-100

6. Calli C, Kitis O, Yunten N, Yurtseven T, Islekel S, Akalin T (2006) Perfusion and diffusion MR imaging in enhancing malignant cerebral tumors. Eur J Radiol 58:394-403 
7. Cao Y, Tsien CI, Nagesh V, Junck L, Ten Haken R, Ross BD et al (2006) Survival prediction in high-grade gliomas by MRI perfusion before and during early stage of RT [corrected]. Int J Radiat Oncol Biol Phys 64:876-885

8. Cha S, Johnson G, Wadghiri YZ, Jin O, Babb J, Zagzag D et al (2003) Dynamic, contrast-enhanced perfusion MRI in mouse gliomas: correlation with histopathology. Magn Reson Med 49:848-855

9. Cha S, Lupo JM, Chen MH, Lamborn KR, McDermott MW, Berger MS et al (2007) Differentiation of glioblastoma multiforme and single brain metastasis by peak height and percentage of signal intensity recovery derived from dynamic susceptibilityweighted contrast-enhanced perfusion MR imaging. AJNR Am J Neuroradiol 28:1078-1084

10. Cha S, Tihan T, Crawford F, Fischbein NJ, Chang S, Bollen A et al (2005) Differentiation of low-grade oligodendrogliomas from low-grade astrocytomas by using quantitative blood-volume measurements derived from dynamic susceptibility contrastenhanced MR imaging. AJNR Am J Neuroradiol 26:266-273

11. Chao ST, Suh JH, Raja S, Lee SY, Barnett G (2001) The sensitivity and specificity of FDG PET in distinguishing recurrent brain tumor from radionecrosis in patients treated with stereotactic radiosurgery. Int J Cancer 96:191-197

12. Chaskis C, Stadnik T, Michotte A, Van Rompaey K, D’Haens J (2006) Prognostic value of perfusion-weighted imaging in brain glioma: a prospective study. Acta Neurochir (Wien) 148:277285

13. Chernov M, Hayashi M, Izawa M, Ochiai T, Usukura M, Abe K et al (2005) Differentiation of the radiation-induced necrosis and tumor recurrence after gamma knife radiosurgery for brain metastases: importance of multi-voxel proton MRS. Minim Invasive Neurosurg 48:228-234

14. Di Costanzo A, Scarabino T, Trojsi F, Giannatempo GM, Popolizio T, Catapano D et al (2006) Multiparametric 3T MR approach to the assessment of cerebral gliomas: tumor extent and malignancy. Neuroradiology 48:622-631

15. Flickinger JC, Kondziolka D, Lunsford LD, Coffey RJ, Goodman ML, Shaw EG et al (1994) A multi-institutional experience with stereotactic radiosurgery for solitary brain metastasis. Int J Radiat Oncol Biol Phys 28:797-802

16. Fuss M, Wenz F, Scholdei R, Essig M, Debus J, Knopp MV et al (2000) Radiation-induced regional cerebral blood volume (rCBV) changes in normal brain and low-grade astrocytomas: quantification and time and dose-dependent occurrence. Int $\mathrm{J}$ Radiat Oncol Biol Phys 48:53-58

17. Henry RG, Vigneron DB, Fischbein NJ, Grant PE, Day MR, Noworolski SM et al (2000) Comparison of relative cerebral blood volume and proton spectroscopy in patients with treated gliomas. AJNR Am J Neuroradiol 21:357-366

18. Huber PE, Hawighorst H, Fuss M, van Kaick G, Wannenmacher MF, Debus J (2001) Transient enlargement of contrast uptake on MRI after linear accelerator (linac) stereotactic radiosurgery for brain metastases. Int J Radiat Oncol Biol Phys 49:1339-1349

19. Kimura T, Sako K, Tanaka K, Gotoh T, Yoshida H, Aburano T et al (2004) Evaluation of the response of metastatic brain tumors to stereotactic radiosurgery by proton magnetic resonance spectroscopy, 201TlCl single-photon emission computerized tomography, and gadolinium-enhanced magnetic resonance imaging. J Neurosurg 100:835-841

20. Knopp EA, Cha S, Johnson G, Mazumdar A, Golfinos JG, Zagzag D et al (1999) Glial neoplasms: dynamic contrast-enhanced T2*weighted MR imaging. Radiology 211:791-798

21. Koichi M (2007) Differential diagnosis of tumor recurrence and radiation necrosis after radiosurgery for brain metastasis with MR perfusion imaging. San Francisco. Ref Type: Conference Proceeding
22. Kondziolka D, Patel A, Lunsford LD, Kassam A, Flickinger JC (1999) Stereotactic radiosurgery plus whole brain radiotherapy versus radiotherapy alone for patients with multiple brain metastases. Int J Radiat Oncol Biol Phys 45:427-434

23. Law M, Cha S, Knopp EA, Johnson G, Arnett J, Litt AW (2002) High-grade gliomas and solitary metastases: differentiation by using perfusion and proton spectroscopic MR imaging. Radiology 222:715-721

24. Law M, Oh S, Babb JS, Wang E, Inglese M, Zagzag D et al (2006) Low-grade gliomas: dynamic susceptibility-weighted contrast-enhanced perfusion MR imaging-prediction of patient clinical response. Radiology 238:658-667

25. Law M, Yang S, Babb JS, Knopp EA, Golfinos JG, Zagzag D et al (2004) Comparison of cerebral blood volume and vascular permeability from dynamic susceptibility contrast-enhanced perfusion MR imaging with glioma grade. AJNR Am J Neuroradiol 25:746-755

26. Law M, Yang S, Wang H, Babb JS, Johnson G, Cha S et al (2003) Glioma grading: sensitivity, specificity, and predictive values of perfusion MR imaging and proton MR spectroscopic imaging compared with conventional MR imaging. AJNR Am J Neuroradiol 24:1989-1998

27. Lee MC, Cha S, Chang SM, Nelson SJ (2005) Dynamic susceptibility contrast perfusion imaging of radiation effects in normal-appearing brain tissue: changes in the first-pass and recirculation phases. J Magn Reson Imaging 21:683-693

28. Lee MC, Cha S, Chang SM, Nelson SJ (2006) Partial-volume model for determining white matter and gray matter cerebral blood volume for analysis of gliomas. J Magn Reson Imaging $23: 257-266$

29. Leimgruber A, Ostermann S, Yeon EJ, Buff E, Maeder PP, Stupp $R$ et al (2006) Perfusion and diffusion MRI of glioblastoma progression in a four-year prospective temozolomide clinical trial. Int J Radiat Oncol Biol Phys 64:869-875

30. Lichy MP, Henze M, Plathow C, Bachert P, Kauczor HU, Schlemmer HP (2004) Metabolic imaging to follow stereotactic radiation of gliomas-the role of $1 \mathrm{H}$ MR spectroscopy in comparison to FDG-PET and IMT-SPECT. Rofo 176:1114-1121

31. Ludemann L, Grieger W, Wurm R, Wust P, Zimmer C (2005) Quantitative measurement of leakage volume and permeability in gliomas, meningiomas and brain metastases with dynamic contrast-enhanced MRI. Magn Reson Imaging 23:833-841

32. Oh J, Henry RG, Pirzkall A, Lu Y, Li X, Catalaa I et al (2004) Survival analysis in patients with glioblastoma multiforme: predictive value of choline-to- $\mathrm{N}$-acetylaspartate index, apparent diffusion coefficient, and relative cerebral blood volume. J Magn Reson Imaging 19:546-554

33. Patchell RA, Tibbs PA, Regine WF, Dempsey RJ, Mohiuddin M, Kryscio RJ et al (1998) Postoperative radiotherapy in the treatment of single metastases to the brain: a randomized trial. JAMA 280:1485-1489

34. Patchell RA, Tibbs PA, Walsh JW, Dempsey RJ, Maruyama Y, Kryscio RJ et al (1990) A randomized trial of surgery in the treatment of single metastases to the brain. $\mathrm{N}$ Engl $\mathrm{J}$ Med 322:494-500

35. Peterson AM, Meltzer CC, Evanson EJ, Flickinger JC, Kondziolka D (1999) MR imaging response of brain metastases after gamma knife stereotactic radiosurgery. Radiology 211:807-814

36. Plotkin M, Eisenacher J, Bruhn H, Wurm R, Michel R, Stockhammer $\mathrm{F}$ et al (2004) 123I-IMT SPECT and 1H MRspectroscopy at $3.0 \mathrm{~T}$ in the differential diagnosis of recurrent or residual gliomas: a comparative study. J Neurooncol 70:49-58

37. Plowman PN (1999) Stereotactic radiosurgery. VIII. The classification of postradiation reactions. Br J Neurosurg 13:256-264

38. Provenzale JM, York G, Moya MG, Parks L, Choma M, Kealey S et al (2006) Correlation of relative permeability and relative 
cerebral blood volume in high-grade cerebral neoplasms. AJR Am J Roentgenol 187:1036-1042

39. Ross DA, Sandler HM, Balter JM, Hayman JA, Archer PG, Auer DL (2002) Imaging changes after stereotactic radiosurgery of primary and secondary malignant brain tumors. J Neurooncol 56:175-181

40. Samnick S, Bader JB, Hellwig D, Moringlane JR, Alexander C, Romeike BF et al (2002) Clinical value of iodine-123-alphamethyl-L-tyrosine single-photon emission tomography in the differential diagnosis of recurrent brain tumor in patients pretreated for glioma at follow-up. J Clin Oncol 20:396-404

41. Schlemmer HP, Bachert P, Henze M, Buslei R, Herfarth KK, Debus $J$ et al (2002) Differentiation of radiation necrosis from tumor progression using proton magnetic resonance spectroscopy. Neuroradiology 44:216-222

42. Schlemmer HP, Bachert P, Herfarth KK, Zuna I, Debus J, van Kaick G (2001) Proton MR spectroscopic evaluation of suspicious brain lesions after stereotactic radiotherapy. AJNR Am J Neuroradiol 22:1316-1324

43. Serizawa T, Saeki N, Higuchi Y, Ono J, Matsuda S, Sato M et al (2005) Diagnostic value of thallium-201 chloride single-photon emission computerized tomography in differentiating tumor recurrence from radiation injury after gamma knife surgery for metastatic brain tumors. J Neurosurg 102(Suppl):266-271

44. Shaw E, Scott C, Souhami L, Dinapoli R, Kline R, Loeffler J et al (2000) Single dose radiosurgical treatment of recurrent previously irradiated primary brain tumors and brain metastases: final report of RTOG protocol 90-05. Int J Radiat Oncol Biol Phys 47:291-298

45. Sonoda Y, Kumabe T, Takahashi T, Shirane R, Yoshimoto T (1998) Clinical usefulness of 11C-MET PET and 201T1 SPECT for differentiation of recurrent glioma from radiation necrosis. Neurol Med Chir (Tokyo) 38:342-347

46. Sugahara T, Korogi Y, Kochi M, Ikushima I, Hirai T, Okuda T et al (1998) Correlation of MR imaging-determined cerebral blood volume maps with histologic and angiographic determination of vascularity of gliomas. AJR Am J Roentgenol 171:1479-1486

47. Sugahara T, Korogi Y, Tomiguchi S, Shigematsu Y, Ikushima I, Kira $\mathrm{T}$ et al (2000) Posttherapeutic intraaxial brain tumor: the value of perfusion-sensitive contrast-enhanced MR imaging for differentiating tumor recurrence from nonneoplastic contrastenhancing tissue. AJNR Am J Neuroradiol 21:901-909

48. Truong MT, St Clair EG, Donahue BR, Rush SC, Miller DC, Formenti SC et al (2006) Results of surgical resection for progression of brain metastases previously treated by gamma knife radiosurgery. Neurosurgery 59:86-97

49. Tsuyuguchi N, Sunada I, Iwai Y, Yamanaka K, Tanaka K, Takami T et al (2003) Methionine positron emission tomography of recurrent metastatic brain tumor and radiation necrosis after stereotactic radiosurgery: is a differential diagnosis possible? J Neurosurg 98:1056-1064

50. Varlotto JM, Flickinger JC, Niranjan A, Bhatnagar A, Kondziolka D, Lunsford LD (2005) The impact of whole-brain radiation therapy on the long-term control and morbidity of patients surviving more than one year after gamma knife radiosurgery for brain metastases. Int $\mathrm{J}$ Radiat Oncol Biol Phys 62:1125-1132

51. Varlotto JM, Flickinger JC, Niranjan A, Bhatnagar AK, Kondziolka D, Lunsford LD (2003) Analysis of tumor control and toxicity in patients who have survived at least one year after radiosurgery for brain metastases. Int J Radiat Oncol Biol Phys $57: 452-464$

52. Weber MA, Lichy MP, Thilmann C, Gunther M, Bachert P, Maudsley AA et al (2003) Monitoring of irradiated brain metastases using MR perfusion imaging and $1 \mathrm{H}$ MR spectroscopy. Radiologe 43:388-395

53. Weber MA, Thilmann C, Lichy MP, Gunther M, Delorme S, Zuna I et al (2004) Assessment of irradiated brain metastases by means of arterial spin-labeling and dynamic susceptibilityweighted contrast-enhanced perfusion MRI: initial results. Invest Radiol 39:277-287

54. Weber MA, Zoubaa S, Schlieter M, Juttler E, Huttner HB, Geletneky K et al (2006) Diagnostic performance of spectroscopic and perfusion MRI for distinction of brain tumors. Neurology 66:1899-1906

55. Wetzel SG, Cha S, Johnson G, Lee P, Law M, Kasow DL et al (2002) Relative cerebral blood volume measurements in intracranial mass lesions: interobserver and intraobserver reproducibility study. Radiology 224:797-803 